\title{
MIS Al - artificial intelligence application in minimally invasive surgery
}

\author{
Zbigniew Nawrat ${ }^{1-3}$ \\ ${ }^{1}$ Chair and Department of Biophysics, Faculty of Medical Sciences in Zabrze, Medical University of Silesia, Zabrze 41-808, \\ Poland. \\ ${ }^{2}$ Professor Zbigniew Religa Foundation of Cardiac Surgery Development, Heart Prostheses Institute, Zabrze 41-800, Poland. \\ ${ }^{3}$ International Society for Medical Robotics, Zabrze 41-800, Poland.
}

Correspondence to: Prof. Zbigniew Nawrat, Department of Biophysics, School of Medicine with the Division of Dentistry in Zabrze, Medical University of Silesia, 19 H. Jordan Str., Zabrze 41-808, Poland. E-mail: znawrat@sum.edu.pl nawrat@frk.pl

How to cite this article: Nawrat Z. MIS AI - artificial intelligence application in minimally invasive surgery. Mini-invasive Surg 2020;4:28. http://dx.doi.org/10.20517/2574-1225.2020.08

Received: 10 Jan 2020 First Decision: 19 Mar 2020 Revised: 30 Mar 2020 Accepted: 23 Apr 2020 Published: 16 May 2020

Science Editor: Noriyoshi Sawabata Copy Editor: Jing-Wen Zhang Production Editor: Tian Zhang

\begin{abstract}
This chapter is devoted towards analyzing the progress and barriers to the development of artificial intelligence (AI) and medical robotics in minimally-invasive surgery. The less invasive the surgical intervention and the further the surgeon is from the operating table, the greater the roles of decision support systems (AI) and performance of specific tasks (by medical robots).
\end{abstract}

Keywords: Artificial intelligence, medical robots, mini-invasive surgery

\section{INTRODUCTION}

The robot is not a machine but an IT device that creates a great opportunity for the integration of the entire diagnostic system with the operator ${ }^{[1]}$. "The future of technology and medicine is not in the blood and bowels at all, but in bits and bytes" ${ }^{2]}$. This is how Prof. Richard Satava, a surgeon from the University of Washington who led the first surgical robot project funded by the DARPA (US Defense Advanced Research Projects Agency), summarizes his many years of experience and visions for the future of surgery.

Surgery is a specific type of medical activity that uses direct physical methods of intervention in a body area damaged by illness or injury. Precise movements by the surgeon requires proper planning and control. Correct positioning and functioning of the tools requires good image quality and all current information from the operating field.

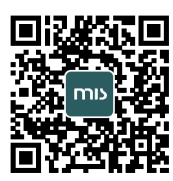


Information is processed during the work of the surgeon or via remote control. The effect of the operation (achievement of the assumed goal) should be measurable and verifiable. Only then can it be used to develop the standard of the service performed, lead to automation and the independence of medical robots in the future.

The operation is part of the patient's treatment strategy. Surgical planning provides a possible and effective approach to use available resources (tools, team, equipment, biological or artificial materials) for the creation of a specific impact (using physical, chemical, biological phenomena) on biological tissues.

The surgeon uses both conditioning and coordination of motor skills to process information during the operation. The information obtained in the process of education and practice (optimized through knowledge and experience), and diagnosis are provided by human senses.

\section{INTELLIGENCE}

Intelligence is the ability of humans to perceive, analyze and adapt to changes in the environment. The ability to understand, learn and use knowledge and skills in different situations, intelligence is a feature of the mind that is responsible for reacting appropriately to and solving problems using: understanding of words, verbal fluency, numerical and spatial abilities, reasoning, memory and perception.

The space we live in is a space of information. For the doctor, the surgical field is also a space of information. If we manage to digitize all images, physicochemical data, and the patient's medical history in the evaluation process, we can analyze this collection of information. If we can digitally describe the treatment methods (the impact of drugs and surgical operations), we can then build a treatment model for a given patient.

Intelligence - both natural and artificial - will be used to optimize the decision-making process for patients in this multidimensional information space.

\section{Artificial inteligence}

In my opinion, artificial intelligence is a part of robotics. The source of the word robot is associated with the figure of an artificial man introduced by the Czech Karel Čapek 100 years ago - R.U.R. Rossumovi Univerzální Roboti (Rossum's Universal Robots). Robotics is a technical discipline, which deals with the synthesis of certain human functions through the use of mechanisms, sensors, executive assemblies and computers. Because humans have a brain, artificial intelligence is an integral part of robotics.

Generally, artificial intelligence was created for communication between machinery and human intelligence. The robot is an artificial man or part of a humanoid robot, artificial organs or artificial intelligence.

Kevin Warwick, known as the first human cyborg (after implanting an electronic interface) claims ${ }^{[3]}$ that "where a brain is involved it must be seen as part of an overall system - adapting to the system's needs". Warwick is a pioneer in studying the connection between the biological body and the robot.

\section{Robots}

The traditional definition of robot means artificial man. From this point of view, the robot should have the ability to move (to perform mechanical work) and make decisions based on information provided by its senses (intelligence). While many animals have these features as well, human beings also have, apart from intelligence (which determines the correct response to stimuli), a reasoning mind, i.e., the ability to use abstract objects (theory) for calculation, objectification, prevention, and planning. Man also has consciousness. 
The number of people in the world is approaching 8 billion and 3 million robots help them in industries, factories and services. According to the $\operatorname{IFR}^{[4]}$ there are 85 and 114 robots per 10,000 employees in the world and Europe respectively. Websites providing various current data measurements for humanity (such as https://www.worldometers.info/pl/) may soon provide information about robots supporting people at work and at home too.

Robots were introduced to factories when there were not enough people to produce the right number of products. And how is it with the surgeons? Accordingly ${ }^{[5]}$, "an insufficient client surgical workforce is a major barrier to safe surgical care for billions of people worldwide". Worldwide, there are an estimated 1,112,727 specialist surgeons and 550,134 anesthesiologists. Low- and lower-middle income countries, representing $48 \%$ of the global population, comprise about $20 \%$ of this workforce. In terms of density, lowincome countries have 0.7 providers per 100,000 population, compared with 5.5 in lower-middle income countries and 56.9 in high-income countries ${ }^{[6]}$.

I believe that many individuals, like me, believe that it is our duty to reduce these differences in access to a good level of medical services. Of course, the biggest role here is in education and investment but perhaps progress in the use of artificial intelligence (AI) in medicine (that is the dissemination of the standard) will provide a real chance to reverse this catastrophic trend of ever-growing disparity in access.

How many operations are performed in the world? Depending on the definition used and access to data, at least 10 million different types of operations are performed each year ${ }^{[7]}$ and this number can even go up to 300 million $^{[8]}$. Based on available data, an estimation of the global volume of surgery using a modeling strategy suggests that "We have estimated that the global volume of major surgery in 2004 was between 187.2 and 281.2 million cases per year, which has substantial implications for public-health planning." ${ }^{[0]}$. The authors' findings suggest that surgery now occurs at a tremendous volume worldwide, in both rich and poor settings. This unprecedented worldwide growth in surgery shows a great need for public-health efforts to improve the monitoring, safety, and availability of surgical services, especially in view of their high risk and expense $\mathrm{e}^{[9]}$.

It is important yet difficult to estimate the real number of operations. There are certainly about 300 million if the authors calculate ${ }^{[9]}$ based on one operated person per 25 living people.

What about standards? How do you count and oversee it? From these examples, we can see that one of the needs that can be met by digital monitoring and AI methods is supervision and access to current data and to analyze them.

Let's return to the robots. Just as robots solved the problem of mass production, perhaps they will allow the dissemination of uniform standards in surgery. They will facilitate access to good medical services. For now, however, while robots increase precision for some medical tasks, they are very expensive; surgeons are helped by about 5000 robots. Every year, almost 1 million minimally-invasive operations using da Vinci robots are performed ${ }^{[10]}$. But these are telemanipulators and every movement of the tool and all decisions are still made by the operator. In addition, thousands of diagnostic robots perform tasks semiautomatically.

Robot control consists of perception, data processing and action. For robots to make a decision, it will need to have access to information and the ability to analyze it. Having information reduces the uncertainty (indeterminacy) of objects or relationships between objects, and allows recognition of the state of the system. Sensors are responsible for obtaining information about the environment and the current state (position) of the robot's components and its system. An important element of service robot control systems is the ability to process video information received from the robot's environment. 
Like humans, the knowledge and skills of robots result from education, experience and usable memory. As with human verification, optimization must be associated with the elimination of wrong decisions and deeds. AI robots can get knowledge from people - this is the first level of learning in robots. If we pass on a collection of information about how it is or how we think it is, then the robot will make decisions that are perhaps burdened with our mistakes. If we teach the robot to read information (measurable data) from sensors and provide algorithms for the formation of correct decisions based on this information, we will achieve an automated device. If we allow it to modify decisions and actions and assess their effects, we will have a self-learning system that can make decisions different from those that we consider as appropriate. Many of us have already been refused a loan in a bank on a similar basis. But, if we give the robot design features based on human, but with additional motion capabilities, we can achieve much better "manual" function than humans. If we give the robot tools and greater efficiency and accessibility to the areas of the human body that we operate on, tissues, cells or genes - then we get a surgical robot with practical skills that are not accessible by humans.

Robots (Cobots) interacting with people change the way many professions perform. Robots create the possibility of standardization and constant improvement of quality through learning (AI) and communication with a professional information network (professional databases and management systems) as well as with other medical devices (diagnostic, therapeutic, rehabilitation), and also elements of hospital infrastructure.

\section{Why do we need artificial intelligence in mini-invasive surgery?}

Man is only as good and useful as his senses allow. Similarly, a robot that is created in the image of a human being, by definition, cannot do more advanced work if it has a limited number of sensors, or lacks the processing of sufficient information to make the right decisions. After all, robots are all about doing work and in surgery, it is all about decision-making and mechanical work.

Suppose, in favor of these considerations, that (1) intelligence is a certain ability to make decisions independently, based on the analysis of signals (senses) that determine the state of the environment and the possibility of the subject's impact (the surgeon using his tools) based on basic knowledge of the entire system (memory) that was developed during the learning process (system evolution by verification and optimization of goal achievement); (2) surgery is an action of removing the effects of a disease, birth defects or injury (bodily injury due to various reasons), the action of mechanically modifying the structure of tissues and organs (surgery) and/or the introduction of natural or artificial elements to replace parts of the body or supporting proper bodily functions (passive or active, artificial and biotechnological implants e.g., stem cells and devices for physical, and mechanical, electrical or chemical stimulation of tissues).

We treat the human body as a biological, physical, chemical and biocybernetic (IT) system. Why is artificial intelligence important in medicine? First of all, decision-making plays a key role in every medical process. Based on the analysis of diagnostic data and medical history, the treatment process begins and its effectiveness is verified in the next step. The basis of evolutionary progress is the process of learning, remembering and disseminating standards. In medicine, the possibility of direct proof is very rare. Usually, the number of unknowns does not allow building full cause-and-effect knowledge to define the possibilities of our therapeutic effects. We do not have the theory of the whole organism, nor do we have the theory of one or another disease, similar to the theories of physics. That is why the doctor makes decisions based on random diagnostic data (and not a full description of the whole organism) and in the treatment specialties such as surgery, the role of sensory assessment (sight, touch, smell, hearing) and manual skills increases (due to the speed of action and real risk). 
If, however, we assume that we would like to operate in a place where our intervention is necessary by methods that reduce the risk of damage to healthy tissues as much as possible, it means the loss of the ability to freely view and touch the tissue, the inability to directly insert our hands in the place of surgery. This is the current MIS dilemma. Is this beneficial for the patient? Can a surgeon possibly do this?

This is a challenge for creating new tools and both artificial intelligence and robots are one of them.

In the mathematical context, artificial intelligence is not an ordinary algorithm, which is a mathematical record of a cause and effect relationship, but a system based on say, neural networks that map the way of building memory pathways in the human brain - i.e., a decision system based on knowledge and experience, similar to the learning process. We do not quite know why such a decision is made but we are sure that the process of system optimization achieved the appropriate level of excellence. This is interesting from the point of view of applying AI successfully in medicine, as it proves the existence of an art factor in the craft of the doctor. You cannot replace a doctor with an automatic machine, i.e., a simple machine. Algorithms alone are likely not enough. There must be artificial intelligence in the decision making process and the robot in execution.

In medicine, artificial intelligence can definitely play a positive role in developing strategies and solutions for operations.

The word "strategy" comes from the Greek words stratós - army, army, and ágein - to command. Carl von Clausewitz defined that strategy is the science of using battles for war purposes. Strategy is not theoretical planning, but it is very close to action, modified by a steady flow of information.

The word "tactics" comes from the Greek words tássein, meaning táttein stack, organize, arrange. The commander creates and effectively uses the elemental system to combat factors such as destruction, movement and information. Isn't it also the essence of surgical intervention?

Surgery is a special human activity related to complex actions for achieving specific purposes. The complexity of an action is the need to assess the condition of the starting issue and making decisions about the distributed action in time divided into roles of the members of a special team.

Operation is a part of the patient's treatment. The doctor develops a treatment strategy and tactics to define the next elements of the procedure. Operation planning includes defining the space and subject of the operation, choosing methods, materials and devices, program of using the operating and accompanying team, and finally, the choice of the sequence of treatments, activities, movements and the impact of tools in the space of operations. The way of conducting surgery significantly depends on the equipment available.

\section{Planning and advisory systems - extended surgeon information space}

The surgeon who operates in a less invasive way has impaired access to information from the patient's body, the surgical field. In the classic version of video-surgery, a very good quality image is obtained - enlarged with clear outlines of contours and colors. We can support this set of visual information by adding information obtained in the diagnostic and analytical process. The ways to visualize this additional information is a separate field of ergonomics, including virtual and augmented reality technology.

In my team, we have been developing the use of computer simulation of surgical operations and the use of physical modeling to improve the patient-specific decision making process for many years [Figure 1]. The skillful use of physicists' knowledge allows us to obtain information, e.g., what will be the flow in a given branch of the coronary vessels after performing a sequential or single bypass, what will be the flow, pressure, regurgitation after implantation of a given type of prosthesis, etc. 

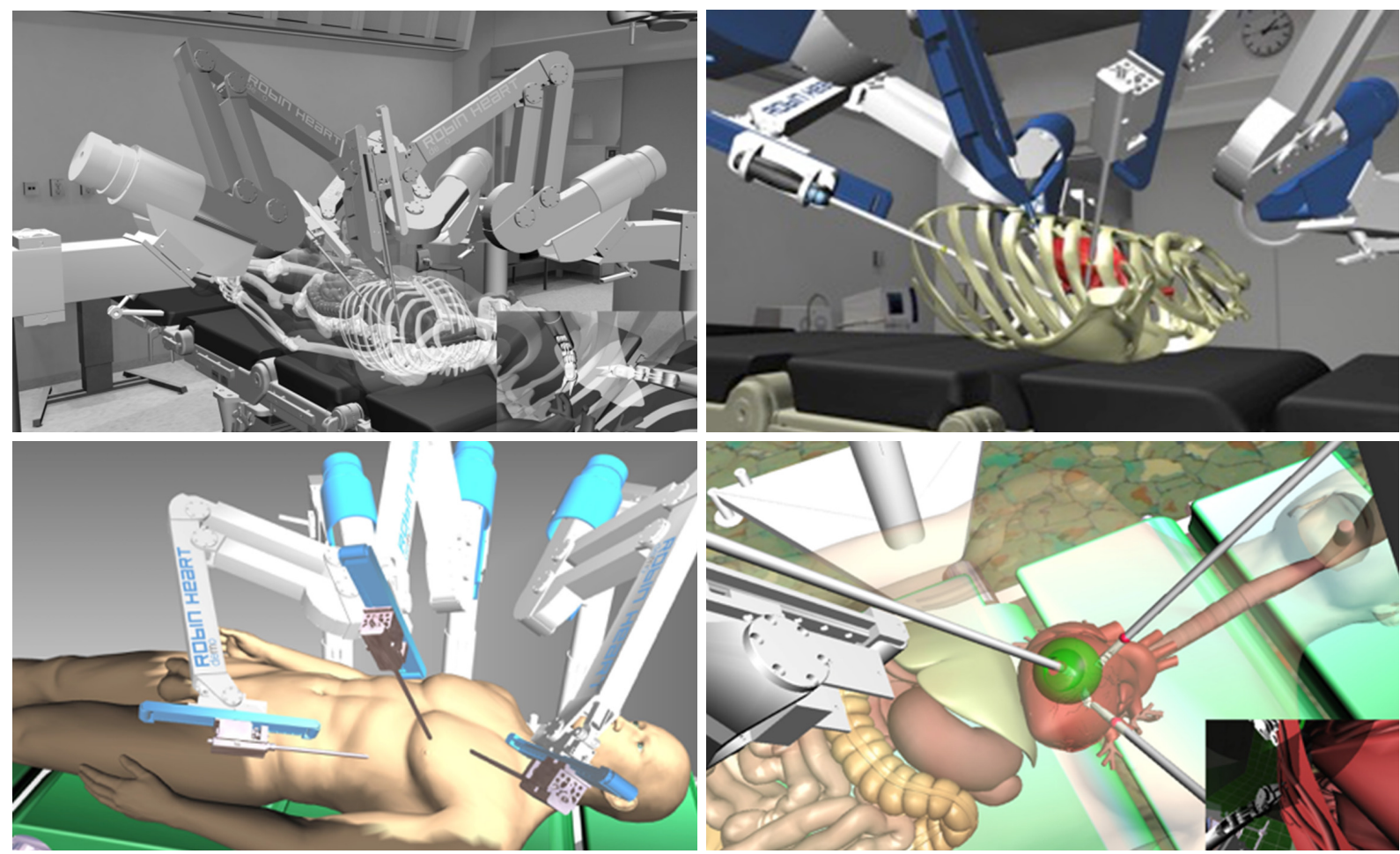

Figure 1. Virtual operating room made in FRK (in a team led by the author). It is used for planning operations using Robin Heart robots (examples are for heart surgery) and for testing robots (in the conceptual phase) and training surgeons. The developed program in virtual space technology was tested for use: (1) on a computer stand; (2) in the Robin Heart Shell 2 robot control console; (3) VR goggles (Oculus). The images below are examples of how to visualize simulated operations. The last image outlines the green work space available for the tool chosen by the surgeon

This means that we can enrich the image with prognostic information. Moreover, if we introduce an image analysis system of the operations performed by a surgeon, we can modify it to keep up with the effects of surgery. This is one of the aims of my research work in my Fundacja Rozwoju Kardiochirurgii (FRK) team.

However, this is still not putting AI into surgery. We have known for many years that we can use telemedicine systems for consulting and verification of the correctness of actions taken by a teacher surgeon who is located away from the operating room. The endoscopic track can be connected to a conference room where all participants can comment live on the operation which is performed in the hospital.

Telemedicine systems are successfully used when, for example, the surgeon has to undertake very little surgery, which is unusual for the practice, but in this way can use the advice directly from a more experienced specialist. In the information exchange system, we thus turn on the brain of another person who does not physically participate directly in the operation. However, if we imagine that we can model online surgery in a digital, computer (simulation) or physical (modeling) system and thus create possibilities for the history of operations by forecasting the results of subsequent steps taken by the doctor, we are then building a very advanced advisory system based on experimental facts (model). If the model is perfect (which is extremely difficult for physiological models) then we will get excellent advice. We can also base our advice for the surgeon on the analysis of real data, from real operations, i.e., Big Data analysis. In this way, we can build a very advanced advisory system based on medical facts. Clearly, this reasoning seeks to demonstrate that the combination of these approaches can be a breakthrough in the quality of advisory systems. The systems supporting the real surgeon while making decisions should graphically indicate the location, place of intervention and provide biological-chemical-physical data that is both current and forecasted based on a specific selection of tactics. 
The decision-making system based on learning from patient databases, controlled and verified in every degree and iteration of its development, was developed by a group of outstanding professionals such as the well-known IBM Watson computer. Unfortunately, its skills, despite the enormous computing power, are still no better than a good doctor. Watson has now obtained approval for decisions regarding selected oncological diseases (by analyzing data from 200,000 correctly identified patients, he can make decisions with an accuracy of up to $97 \%$, but only for a few diseases for now). However, Watson's difficulties indicate that entering into a live partnership during operations is still a long way off. Artificial intelligence can contribute to reducing errors, improving standards and the quality of performed operations, and increasing patient safety.

1. Indirectly, passively as the basis for the work of the advisory system when planning and performing operations.

2. Indirectly - as an element of surgical telemanipulator or mechatronic tools.

3. Directly - as the basis for the operation of an autonomous surgical robot.

Improving the decision making process is always associated with access to good, reliable and timely information. The surgeon manually performing a classic operation uses information from all his senses. The surgeon loses access to all this information by moving away from the patient during surgery. For instance, in endoscopic instruments with a distance of about half a meter and with telemanipulators over a distance of several meters or many kilometers.

\section{Medical robots}

The segment of medical robot systems (in market analyses) is divided into surgical robot systems, rehabilitation robots, non-invasive radio surgical robots, hospital and pharmacy robots and other medical robot systems. The following companies are present on the market: Intuitive Surgical, Inc. (USA), Stryker Corporation (USA), Mazor Robotics Ltd. (Israel), Hocoma AG (Switzerland), Hansen Medical Inc. (USA), Accuray Incorporated (USA), Omnicell, Inc. (USA), Ekso Bionics Holdings, Inc. (USA), ARxIUM (USA), Kirby Lester LLC (USA), Houston Medical Robotics (USA), Otto Bock Healthcare, Kinova robotics, Varian Medical Systems, Hocoma AG, Vecna Robotics, Globus Medical, IRobot Corporation, Titan Medical, Inc., and KB Medical SA.

Medical robotics has been the most successful so far in the field of surgical and rehabilitation robots (including exoskeletons). The robot leader on the soft tissue telemanipulator market is the da Vinci robot (Intuitive Surgical). Five thousand robots are currently used in approximately 1 million operations per year, mainly urological and gynecological. New surgical robots appear, e.g., ALF-X (USA), Titan (Canada), and in other areas such as Virtual Incision, TransEnterix (SurgiBot, Senhence robot-assisted surgical system), Coridus Vascular Robotics (cardiological robot, CorPath GRX) or Artas (robot platform for hair implantation) and endoscopic Monarch (Auris Health) and orthopedic Renaissance (Mazor Robotics). Medtronic introduced surgical HUGO (Einstein), and Medrobotics - FLEX - flexible endoscopic robotic tools.

Corindus, producer of CorPath GRX platform, received permission from the United States Food and Drug Administration (FDA) for the first automated robot movement called "Rotate on Retract (RoR)" in $2018^{[11]}$. This is the first step towards introducing autonomous robot operations.

Telemaniplators allow remote-controlled operations from different distances. However, there is a distance-dependent delay in transmission of operation images to the surgeon that can be dangerous. Most researchers consider $300 \mathrm{~ms}$ as the limit in delay time $\mathrm{e}^{[12]}$. The implementation of $5 \mathrm{G}$ technology will overcome barriers and reduce the delay of $0.27 \mathrm{~s}$ to $0.01 \mathrm{~s}$ and also improve image quality ${ }^{[13]}$. Worldwide, about 143 million surgical procedures are not performed due to the lack of knowledge of 
specific procedures ${ }^{[5]}$. Creating a network in which surgical operations are possible at a distance is a technological and political challenge. But only in this way we will then be able to solve the problem of "equal opportunities" for access to appropriate quality medical services.

\section{Autonomy - independence of medical robots}

For medical robots to play their role fully in the face of challenges from the growing needs of services, and the need to increase their quality, they must soon become independent. Stand-alone robots should operate in an information network, which allows access to all information needed to optimize operations in every situation. In history, medical robotics has already received support, several times, from technologies that were perfected for performing industrial tasks, aerospace or military projects. This time, the growing interest of the industry in co-robots - robots cooperating with employees on production lines - and strategies for the development of automation in the field of autonomous vehicles will give appropriate acceleration for the new projects of medical robots. Autonomous vehicles are currently being developed by technology giants such as Google, Apple, Tesla and Uber, a number of automotive companies including Mercedes, Volkswagen/Audi, BMW, General Motors, Volvo, Ford, a consortium of Renault-NissanMitsubishi and Toyota, and companies producing computer components like Nvidia and Intel.

We probably will not make the mistake of systematizing the autonomous capabilities of medical robots based on the five-level classification of autonomous vehicles introduced by the SAE International (Society of Automotive Engineers) standardization organization. The autonomy of medical robots on a five-point scale (modeled on the SAE J3016 standard) though, with the lowest level, 0, is a lack of autonomy:

1. Level 1 is telemanipulation (remote control) with support. In this type of robots, some elements have been introduced to support operations automatically. It can be, for example, an emergency stop system for a robot in a hazardous situation. Surgical robots such as da Vinci and Robin Heart are currently in this group.

2. Level 2 represents robots with the option of partly automated work. A robot that can perform one of the tasks in an automated manner, e.g., tying a node or orientation of the cam-vision track to a tool.

3. Level 3 is highly automated work. The system moves independently in the work space and scope of tasks but is still able to assess the limits of its freedom. If it judges that the working conditions are outside the defined area, the operator must immediately take control of the robot. In the absence of such a reaction, the robot stops. Such robots are self-propelled robots for tele-presentation and socalled robotic nurses for communication and transport of various products and materials in hospitals.

4. Level 4 is fully automated work. The robot works independently but should still be supervised, e.g., by a doctor, rescuer or physiotherapist. An example of a vehicle - a robot - included in this level is the autonomous Volvo XC90 used in Uber tests (in the vehicle there is a driver - a human verification element that can take over steering, after warnings from the control system). Such machines are currently computer tomography or robotic radio-surgical knives that move and operate in accordance with the planned trajectory and tasks specified before the surgery.

5. Level 5 means a robot working fully autonomously. The medical robot works independently, sharing space with the patient and medical staff, makes independent decisions and performs tasks provided for in its specialization. The robot has no tele-manipulation system. An example is the city car prototype developed by Google, Waymo Firefly i.e., a car without a manual control system, including the steering wheel, gear lever or pedals. There are currently no such medical robots.

\section{FRK OWN EXPERIENCE}

The Professor Zbigniew Religa Foundation for Cardiac Surgery Development in Zabrze is a pioneer in Poland in the field of medical robots for heart prostheses and surgery. Robin Heart is the name of the whole family of Polish surgical robots intended for heart surgery (also soft tissue) and for now, consists of: Robin Heart 0, Robin Heart 1 and Robin Heart 2 created in 2000-2003; Robin Heart Vision in 2007- 
2008 (for video surgery, one arm robot); modular robot Robin Heart $\mathrm{mc}^{2}$ from 2009; TeleRobin form 2014 (new solution of the tool platform), followed by subsequent versions of the video surgery robot; ultra light Pelikan and Robin Heart PortVisionAble in the preclinical version. At that time, Robin Heart $\mathrm{mc}^{2}$ was the largest surgical robot in the world. It consists of three arms: two arms working as an assistant and a middle arm equipped with a tool platform (two tools of the main surgeon and vision endoscope). Robin Heart Shell console is equipped with a 3D monitor and an additional touch screen to change control parameters (tremor removal or motion scaling) and an advisory system (that shows the necessary diagnostic data as well as the results of planning and simulating operations). Robin Heart $\mathrm{mc}^{2}$ is a modular robot with the tools, which can be quickly removed from the robot arm and used as manually controlled mechatronic tools. The Robin Heart system was tested in animal experiments at the Experimental Medicine Center of the Medical University of Silesia in 2009-2010 (gallbladder surgery, coronary artery bypass grafting and heart valve repair) ${ }^{[14]}$.

The surgery planning system is based on the use of an original virtual operating room. The simulation of operations was prepared by the team at the Biocybernetics Laboratory to allow the introduction of physical features (pressure and blood flow) to hemodynamic models of heart surgery. The Polish team is preparing to introduce robots to the market, for clinical implementation. Elements of artificial intelligence will, in the first version, be applied to the advisory program presented online when controlling the robot from the Robin Heart Shell console. Subsequently, semi-automatically performed tasks and selected elements of the procedure (e.g., tying the knot while sewing or cutting a hole of a certain shape) will be introduced. The key to introducing AI to surgical robotics are sensors, image analysis and processor speed. We believe that in the future, these robots will be completely independent.

Purposes and necessary conditions for using AI in MIS:

1. In advisory programs and planning support systems, AI serves as a method of linking the current situation during a surgical operation with the results obtained earlier in clinics (if they are digitized, i.e. form elements of the Big Data base) or obtained from physical modeling, computer simulations or the use of models theoretical in calculations.

2. In mechatronic surgical instruments, if the instruments are equipped with sensors (determining the features of local surgical intervention), soft \& harware to understand what specific signals mean for the task being performed and monitors presenting data to the surgeon online.

3. In surgical telemanipulators, if robots are equipped with sensors (determining the features of local surgical intervention and force feedback), AI can be used in a system of supervision and control of performed surgical tasks and creating the basis for working with the image of augmented reality technoology. Innovative surgical instruments equipped with force sensors and/or performing semiautomatic tasks (such as sewing) play a key role in this process.

4. In independent surgical robots, if robots can on-line process information available from sensors, their own computer and cloud database, creating information loop containing physical/chemical/biological data, data from memory resources regarding the practice and theory of selected surgical procedures (imitating the medical knowledge of the surgeon), as a element of central decision making system (AI) and the implementation of surgical tasks.

Of course, we must remember that surgery is collective work, and the team consists of an anesthesiologist and many other specialties including nursing and technical support. There is also information about robots being developed to replace the work of people from the team accompanying the surgeon. It is possible that the entire operation will be robotic. Contrary to common belief, this is not an impossible task, if we were to seriously consider plans to build settlements on the moon or Mars by comparison.

In my Robin Heart Team in Zabrze, we conduct research and design work, and we build and test mechatronic robots and tools. The FRK Biocybernetics Laboratory is also a pioneer in the field of 

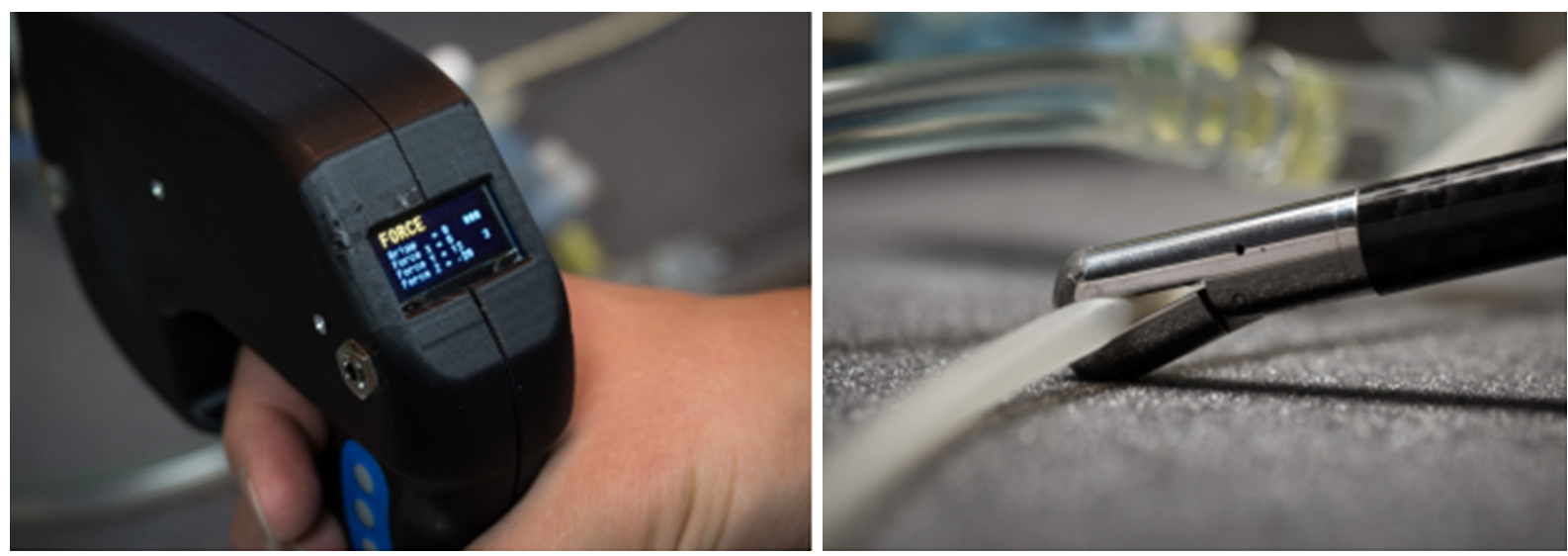

Figure 2. Selected elements of the history of the Robin Heart surgical robot, which was creat-ed in the FRK in a team led by the author

simulation and modeling of heart surgery by creating data on the hemodynamic effects of surgery performed in a given way ${ }^{[15-19]}$. Figure 2 shows examples that demonstrate the achievements of the FRK within the scope of 1-3 of the AI implementation plan for MIS.

\section{Al and ethics}

Text based on ${ }^{[20]}$ The Ethics of Artificial Organs in Implant Expert by Zbigniew Nawrat, with permission.

I believe ethics to be the art of making the right choices. And modern medicine, with its new medical techniques, healthcare systems and financing schemes, creates a completely new area of insecurity in this regard. For ages, philosophers have been analyzing issues connected with man- $v$ s.-man and man- $v s$.-the world relations in order to help us comprehend the reality and find the correct conduct.

The term "ethics" comes from the Greek word ethicos, meaning a way of conduct accepted in society, a conduct according to the legal character (ethoscharacter). Today, "ethics" is colloquially understood as "morality", although the Latin word moralis denotes judgement of the appropriateness of a given action, more so than a person's character.

The use of $\mathrm{AI}$ and robotics in the treatment process facilitates remote medical care, consultations and the monitoring of a patient's condition. It is therefore a chance for greater availability and the quality of medical services. AI and robots are a breakthrough in infrastructure, organization of the operating theatre, and in the specialist training of surgeons. But how do we evaluate a wrong decision or action of a doctor from a distance? How do we divide the responsibility for mistakes of remote robotic devices? The access to information depends on technical resources such as software, etc. Therefore, the final effect is influenced by a number of people - engineers, administrators and economists, etc. as well as fortuitous events. Ethics and morality assume human-to-human contact. Our conscience and empathy work differently in the absence of a direct connection between our actions and their effect on others.

The technological progress in biomedical engineering and regenerative medicine constitute the basis for innovative artificial organs which are adequately small, efficient, durable and energetically and mechanically functional.

A new generation of Micro-Electro-Mechanical Systems is entering medicine. The fast development of bioelectromechanical microsystems (bioMEMS), micropumps and bioinformatics has created new possibilities such as the "lab on a chip" micro-laboratory, which are revolutionizing diagnostics and therapy. 
In the near future, technical devices, entirely artificial organs and robots will be used to secure tissue therapy and genetic therapy. The integration of engineering and biology is a fact.

Thanks to bioengineering and medicine, today we can influence the survival of certain individuals and entire groups of patients.

With the harmonious development of technical and biological sciences and their reasonable implementation, and the quality of life on Earth.

Artificial intelligence is part of this evolution. Since it is an element of management at the level of organs and organisms, such medical procedures on the body will require hospital or state management and we must introduce ethical criteria agreed by all people.

\section{Al - towards greater efficiency, permanent improvement and credibility}

The popularization of telecommunications and the Internet is a good example of democratization and equal access to human achievements. We all can, rich or poor, in America and Africa, in the city or in the countryside, have access to Google search engines and data collected in public libraries around the world. Just as we can check the weather, because we supervise data from various places around the world, we will be able to supervise our body condition - as long as we are able to collect relevant data from all organs of our body. This is the future of "lab on chip". But today, by collecting data from our health records, we can make better decisions about caring for our health. By analyzing diagnostic data, we can make better decisions about invasive medical intervention, but for now the mediator is a person - the doctor. Soon, however, we will be giving the next decision to robots (communication of devices without the mediation of a human as a decision maker) and no one is surprised today when the door in front of us opens automatically. I am convinced that it will be a good time for humans, their health and safety, and we will be able to develop our skills and activities in completely new directions.

In the case of legal responsibility, it is worth looking for an analogy to obtain adequate social support. Computer advisory programs, decision systems and autonomous robots operate either on 1) the basis of transparent algorithms linking the cause and observation with effect and decision, or 2) on the basis of machine and deep learning - AI. In the former, we can conclude that the rules of operation are legible to the professional user (e.g., doctor) and the beneficiary, the client (e.g., patient) and the right of informed consent can be introduced. If the system of performing tasks, producing a council or a specific medical act does not take into account the absolute safety of the patient but other factors like economics (e.g., whether the client has paid for the service or is insured) then it is a matter of rules that we know perfectly well from the operation of health services. If someone introduces a virus and changes the system in a way that poses a danger, we already have (?) ways that are working such as criminal law. However, if the system works on the basis of machine learning, it is a completely new type of relationship where the client is the robot. First, we have an analogy: generally, neither the teacher nor the professor or the father is responsible for the student's deeds although in each case they are in a teacher-student relationship. Similar to the teaching process of students by introducing a different learning system, we can obtain a good or bad AI program/ robot. It can make right or wrong decisions based on the data and save or kill a patient. No one is able to check how it works without starting the system because there are no readable rules inside. Just like looking into the human brain, by itself it is difficult (?) to assess whether it belongs to a surgeon or a shopkeeper. Our only option, as well as the system of training doctors and their acquisition of specialties entitling practice of the profession, will be to test and assess the effectiveness of autonomous robots, and to make sure that no computer virus destroys learning outcomes! Then we free robots from responsibility, just as we free specialists from responsibility, believing that he does the best, in a world where the end result is never obvious and certain. Just like man, AI does not have all the data to "solve the equation of life" for the patient. 
The robot has the right to break. It is not allowed to make a move without the consent of the operator. During power outages and other problems, it must be possible to remove the tools from the patient by means of a medical team. I myself was a witness to this in the early phase of implementation of the da Vinci robot: during an on-line operation for its participants at a conference in Leipzig, one of the arms of the da Vinci robot stopped listening to the surgeon's hand. Our excellent colleague continued his classic surgery without squinting. Experience and skills, reason and knowledge always promote happiness.

Excessive optimism related to the shorter path of gaining experience in robotic operations was the reason for many crisis situations and simple mistakes. The patient's death during the first mitral valve surgery with a robot in the UK was resounding. The surgeon lost visibility after damage to the aorta which flooded the camera. The patient died. "An inquest has heard how Sukumaran Nair lobbied to be allowed to perform a mitral valve repair using a state-of-the art Da Vinci robot, despite performing slowly during non-robotic operations and passing up opportunities to practice on the machine, ${ }^{\text {,21] }}$.

The da Vinci robot is a telemanipulator. The control system allows you to direct the movement of the tools using the movement of the hand of the surgeon sitting at the console. Sometimes the control signal transmission system (e.g., cable damage) or movement mechanism (e.g., mechanical damage) fails. Here is an example of the description of such an event noted by the FDA: "System error codes \#20009 and \#21009 appear when the da Vinci safety system determines a differential change in the angular position of one or more robotic joints on the specified manipulator, as measured by that joint's primary control sensor encoder and the secondary sensor potentiometer. The system alarm system generated fault codefunctioned as designed and there was no injury to the pt. Upon determining this condition, the safety system put da vinci in a "recoverable safe state"[22].

The FDA has an online database MAUDE (Manufacturer and User Device Experience) filed by manufacturers, health care facilities, patients and lawyer, and includes thousands of incidents (ranging from error code bugs to patient deaths) related to the use of various da Vinci robotic systems. In Homa Alemzadeh and colleagues "A Retrospective Study of 14 Years of FDA Data" summarized that between 2000 and 2013, a total of 144 deaths, 1391 patient injuries and 8061 device malfunctions were reported with robotic surgery (10,624 reports at a time when 1.75 million robotic procedures were performed). Device and instrument malfunctions, such as falling of burnt/broken pieces of instruments into the patient (14.7\%), electrical arcing of instruments (10.5\%), unintended operation of instruments (8.6\%), system errors (5\%), and video/imaging problems $(2.6 \%)$, constituted a major part of the reports. Device malfunctions impacted patients in terms of injuries or procedure interruptions. In $1104(10.4 \%)$ of all the events, the procedure was interrupted to restart the system (3.1\%), convert the procedure to non-robotic techniques (7.3\%), or to reschedule it $(2.5 \%)^{[23]}$.

According to the FDA database, in the 5-year period ending on Aug 31, 2017, the agency received 30 reports of incidents in which the patient died in connection with surgery using the da Vinci system. The system did not necessarily cause those deaths, but they occurred after or during surgery in which surgeons used the system. During the same time period, the FDA received 282 reports involving patient injury.

Experts say that the main contributing factors to robotic surgery adverse-event reports are: device failure (30\%), device operational/setup (25\%), user error (20\%), inadequate training (7\%), maintenance issue (7\%) and others $(11 \%)^{[24]}$.

That's all for the manipulator. The equipment or person may fail. But if we were to have an autonomous robot, who or what will be considered guilty? Who will be responsible for the wrong diagnosis or bad result from surgery? Engineer, constructor, director (owner) of the hospital, the supervising doctor or 
even, the economist? Or maybe a lawyer? Or perhaps the family/patient agreeing to use it? Again, looking at the successes and progress (including failures) of the development of autonomous vehicles, we see that the solution will not be easy. It will probably be different for different treatments and activities, depending on the patient's balance of risk and chance. Just as we agree, as road users, that the fire brigade should not follow common traffic rules (as a delaying factor) in order to rescue people. But before we worry about how to solve this problem for interventional medicine, we need to solve this problem for better-developed artificial intelligence applications in diagnostics. Already today, there are companies, for example, which diagnose on the basis of an image analysis of the cornea of the eye and bravely take full responsibility for the opinions issued. Of course, none of these areas of diagnostic imaging (using humans or robots) is 100\% correct.

\section{CONCLUSION}

There is one more important reason why we have to rely on artificial intelligence: human intelligence is decreasing. Humanity has reached a point where successive generations are becoming less and less intelligent. After analyzing over 730 thousand intelligence tests, Norwegian scientists ${ }^{[25]}$ found that, the average IQ in society is now statistically decreasing by 7 points for each generation since the 20th century.

I explain it this way. Increasingly, we are using memory and calculating and decision-making abilities in an easier world full of computers, search engines (Google), smartphones and smartwatches. Convenience comes at the expense of efficiency.

Since human intelligence is diminishing and sooner or later, artificial intelligence will be growing more, many scientists estimate that in twenty years, robots will have human level intelligence. Whether the performance efficiency then will be similar to that of the best surgeons is difficult to say but in general, mechanics does not develop as quickly as electronics. The art of robotics is based on an intelligent combination of mechanical work and information management obtained by sensors.

For now, information about the progress of AI in medicine is not as optimistic as we had expected. Afterall $^{[26]}$, Medical AI, which pulled in $\$ 1.6$ billion in venture capital funding in the third quarter alone, is "nearly at the peak of inflated expectations". Even Topol, the author of "Deep Medicine: How Artificial Intelligence Can Make Healthcare Human Again", also acknowledges that many AI products are "little more than hot air".

As the summarized IBM Watson state of art now says ${ }^{[27]}$, "They've been trying to go into all sorts of things with mixed success and one of the most hopeful things was that they would be able to revolutionize medical care, health care. And it's not worked because they could look up symptoms of various diseases and they could look up cures for various diseases and they could look up medical articles. But they don't understand which are more meaningful than others, which medical articles are reasonable and which are bull and so a lot of doctors have become disillusioned with Watson. And a lot of hospitals have literally pulled the plug".

However, there are fields of medicine, e.g., dermatology ${ }^{[28]}$ and radiology, in which we are already successfully using expert programs. "The test, sold as IDx-DR, screens patients for diabetic retinopathy, a leading cause of blindness, and refers high-risk patients to eye specialists, who make a definitive diagnosis. IDx-DR is the first 'autonomous' AI product - one that can make a screening decision without a doctor. The company is now installing it in primary care clinics and grocery stores ${ }^{\text {"[26] }}$.

So why are the successes of artificial intelligence much better in image recognition? Why did deep learning methods and currently available computing power make AI practical and useful in medicine, now? The 
expert systems (such as MYCIN for the recognition of pathogenic bacteria) were developed to solve medical diagnoses using rules from 50 years ago. For the operation of such a system, all facts and rules, as well as symptoms and histories of patients' diseases should be collected from specialists. Then, the software author needs to enter the data into the computer to conduct "reasoning in accordance with the laws of logic" and to constantly update the system with new data. These systems do not "scale out" and cannot succeed because of a high level of complication of a problem (e.g., starting in 1984 project CYC - sum of all data on human judgments). Only research on the human brain, discoveries explaining the actions of neurons (e.g., Alan Hodgkin, Andrew Huxlay, Bernard Katz) and attempts to transfer this biological knowledge to computer science have led to adequate progress. A good example is one of the deep learning pioneers Terrence Sejnowski, whose achievements can be found in both human brain research and the creation of an artificial brain on this basis ${ }^{[29-33]}$. Advances in machine vision were made when the focus was on the characteristics of the objects rather than on individual pixels. Deep learning is therefore effective for solving problems of image analysis because its structure and operation was based on the analysis of the process of vision and image recognition by the human brain. Fortunately, it took much less time than the evolution for creating our species.

What's more, all doctors are already looking forward to the possibility of using navigation, similar to the ones Google has provided in our cars. As we all remember, the development of car navigation was preceded by creating accurate maps. The diagnostics offered by robots are approaching us soon to achieve such accuracy that will enable similar outcomes in medicine. The first is orthopedics and neurosurgery for which we do not require updates in the millisecond time mode. And the first robot tools that used it with great success were the radiosurgical knives of oncological robots (CyberKnife).

AI and robotics are transforming medical services. We note successes in analyzing medical images (histopathology) and building a knowledge base. In 2017, a Chinese robot called Xiao Yi, developed by Tsinghua University passed China's National Medical Licensing Examination. The robot scored 456 points in the test which was well above the passing mark of $360^{[34]}$. OK. So we now have AI with the knowledge of a medical student, but do we have an experienced doctor?

There are several FDA-approved devices and platforms for robotic surgery and these include the da Vinci Surgical System, Sensei X Robotic Catheter System, FreeHand 1.2 and invendoscopy E200 system. Also approved are Flex ${ }^{\odot}$ Robotic System, Senhance, ARES, the Single-Port Instrument Delivery Extended Research (SPIDER) and the NeoGuide Colonoscope. Other technology platforms waiting for FDA approval include MiroSurge, ViaCath System, SPORT ${ }^{\mathrm{TM}}$ Surgical System, SurgiBot, Versius Robotic System, Master and Slave Transluminal Endoscopic Robot, Verb Surgical, Miniature In Vivo Robot, and the Einstein Surgical Robot ${ }^{[35]}$.

We hope that our robot Robin Heart will soon join this group. Surgical robots are a way to introduce standardization and reduce invasiveness, while ensuring proper operation safety.

"In the future, robotic surgeons will be more involved in the healthcare requirements of individuals. Robots require a communication link and applications that connect the robots to their clients or users. These communication links are usually supported through client/server network connections. Therefore, the networking system is vulnerable to cyber-attacks and consequently, the security and privacy of the robotic platforms is paramount ${ }^{\text {"35] }}$.

In general, robots will get smarter until they finally become necessary. Despite the problems (temporary, in the category of the time of development of our civilization), artificial intelligence and robots are part of the evolution of humanity and medicine. 


\section{DECLARATIONS}

\section{Acknowledgments}

Robin Heart Project was supported by KBN 8 T11E 00118 and projects: PW-004/ITE/02/2004, R1303301 and R13 0058 06/2009, and NCBR - the national Centre for Research and Development - grants: R1303301 and R13 0058 06/2009, Robin PVA - no 178576,TeleRobin - no 181019 and many sponsors. The project of flexible tool supported in part by the European Commission within the STIFF-FLOP FP7 European project FP7/ICT-2011-7-287728 and grants of the ENIAC "INCITE" project No.621278 supported the force-feedback achievements. Currently the author is a partner of Digital Innovation Hubs in Healthcare Robotics HERO H2020 /825003.

\section{Authors' contributions}

The author contributed solely to the article.

\section{Availability of data and materials}

Not applicable.

\section{Financial support and sponsorship}

This work was done as part of my duties to disseminate knowledge about the possibilities offered by robot technologies for medicine supported by the EU project Digital Innovation Hubs in Healthcare Robotics HERO H2020/825003.

\section{Conflicts of interest}

The author declared that there are no conflicts of interest.

\section{Ethical approval and consent to participate}

Not applicable.

\section{Consent for publication}

Not applicable.

\section{Copyright}

(C) The Author(s) 2020.

\section{REFERENCES}

1. Satava RM. Future Direction in Robotic Surgery. In: Rosen J, Hannaford B, Satava RM. editors. Surgical Robotics. System application and Visions. Springer New York, Dordrecht, Heidelberg, London; 2011.

2. Sant'Anna RT, Prates PRL, Sant'Anna JRM, Prates PR, Kalil RAK, et al. Robotic systems in cardiovascular surgery. Rev Bras Cir Cardiovasc 2004;19. Available form: http://www.scielo.br/scielo.php?pid=S0102-76382004000200012\&script=sci_arttext\&tlng=en [Last accessed on 28 Apr 2020]

3. Warwick K. The diminishing human-machine interface. Medical Robotics Reports, International Society for Medical Robotics (ISMR), Zabrze, 2. 2013. pp. 4-11.

4. Executive Summary World Robotics 2019 Industrial Robots. Available form: http://www.ifr.org [Last accessed on 28 Apr 2020]

5. Meara JG, Leather AJM, Hagander L, Alkire BC, Alonso N, et al. Global Surgery 2030: evidence and solutions for achieving health, welfare, and economic development. Lancet 2015;386:569-624.

6. Holmer H, Lantz A, Kunjumen T, Samuel F, Marguerite H et al, Global distribution of surgeons, anaesthesiologists, and obstetricians. The Lancet Global Health 2015;3:S9-S11.

7. Available form: https:/gateway.euro.who.int/en/indicators/hfa_539-6031-total-number-of-inpatient-surgical-procedures-per-year/ visualizations/?EU_MEMBERS;EU_BEFORE_MAY2004;EU_AFTER_MAY2004;CIS;CARINFONET;SEEHN;NORDIC;SMALL\# id $=19634$.

8. Weiser TG, Regenbogen SE, Thompson KD, Haynes AB, Lipsitz SR, et al. An estimation of the global volume of surgery: a modelling strategy based on available data. Available form: https://www.thelancet.com [Last accessed on 28 Apr 2020]

9. Weiser TG, Regenbogen SE, Thompson KD, Haynes AB, Lipsitz SR, et al. An estimation of the global volume of surgery: a modelling 
strategy based on available data. Lancet 2008;372:139-44.

10. Available form: https://www.intuitive.com/en-us/-/media/Project/Intuitive-surgical/files/pdf/2019-intuitive-sustainability-report.pdf?la=en \&hash=CF15F93D4B9FA67710F445500CF3F9BA [Last accessed on 28 Apr 2020]

11. Corindus CorPath GRX surgical robot receives clearance in Japan. Available form: https://www.therobotreport.com/corindus-corpath-grxclearance-japan [Last accessed on 28 Apr 2020]

12. Anvari M, Broderick T, Stein H, Chapman T, Ghodoussi M, et al. The impact of latency on surgical precision and task completion during robotic-assisted remote telepresence surgery. Comput Aided Surg 2005;10:93-9.

13. HealthManagement. Antonio de Lacy $5 \mathrm{G}$ opens the future of telesurgery. Available form: https://healthmanagement.org/c/ healthmanagement/issuearticle/5g-opens-the-future-of-telesurgery [Last accessed on $28 \mathrm{Apr} 2020$ ]

14. Nawrat Z. Robot chirurgiczny - projekty, prototypy, badania, perspektywy. Rozprawa habilitacyjna, Śląski Uniwersytet Medyczny, Katowice 2011.

15. Nawrat Z, Koźlak M. Robin Heart system modelling and training in virtual reality. J Automat Mob Rob Intel Syst 2007;1:62-6.

16. Nawrat Z, Rohr K, Fürjes P, Mucha $\mathrm{L}$, Lis K, et al. Force feedback control system dedicated for robin heart surgical robot. Procedia Engineering 2016;168:185-8.

17. Małota Z, Nawrat Z, Sadowski W. Banchmarking for surgery similators in soft and stiffness-controllable robotics solutions for minimally invasive surgery: the STIFF-FLOP approach. In: Konstantinova J, Wurdemann H, Shafti A, Shiva A, Althoefer K, editors. England: River Publishers Series in Automation, control and Robotics.

18. Mucha $€$, Lis K, Lehrich K, Nawra Z. RobinHand haptic device; soft and stiffness-controllable robotics solutions for minimally invasive surgery: the STIFF-FLOP approach. In: Konstantinova J, Wurdemann H, Shafti A, Shiva A, Althoefer K, editors. Anglia: River Publishers Series in Automation, control and Robotics; 2018. pp. 289-305.

19. Nawrat Z, Mucha $€$, Lis K, Lehrich K, Rohr K, et al. Robot chirurgiczny robin heart tele - następca RobinHeart mc ${ }^{2}$. Med Robotics Rep 2016;5:27-33.

20. Nawrat Z. The ethics of artificial organs. M-Studio, Zabrze: ImplantExpert ed Zbigniew Nawrat Zabrze 2011. pp. 127-35.

21. 'Slow' heart surgeon failed to inform fatal patient he was at greater risk from pioneering robot procedure. Available form: https://www. telegraph.co.uk/news/2018/11/06/slow-heart-surgeon-failed-inform-fatal-patient-greater-risk/ [Last accessed on 28 Apr 2020]

22. Maude adverse event report: intuitive surgical davinci surgical robot. Available form: https://www.accessdata.fda.gov/scripts/cdrh/cfdocs/ cfmaude/detail.cfm?mdrfoi_id=1660340 [Last accessed on 28 Apr 2020]

23. Alemzadeh H, Raman J, Lveson N, Kalbarczyk Z, Lyer RK. Adverse event in robotic surgery: a retrospective study of 14 years of FDA data. PLoS One 2016;11:e151470.

24. da Vinci Robotic Surgery Complications. Available form: https://www.drugwatch.com/davinci-surgery/complications/ [Last accessed on 28 Apr 2020]

25. Bratsberg B, Rogeberg O. Flynn effect and its reversal are both environmentally caused. Proc Natl Acad Sci U S A 2018;115:6674-8.

26. Artificial intelligence is rushing into patient care—and could raise risks. Available form: https://www.scientificamerican.com/article/ artificial-intelligence-is-rushing-into-patient-care-and-could-raise-risks/ [Last accessed on $28 \mathrm{Apr} 2020$ ]

27. Why was ibm watson a flop in medicine? Available form: https://mindmatters.ai/2019/08/why-was-ibm-watson-a-flop-in-medicine/ [Last accessed on 28 Apr 2020]

28. Esteva A, Kupre B, Novoa RA, Ko J, Swetter SM, et al. Dermatologist-level classification of skin cancer with deep neural networks. Nature 2017;542:115-8.

29. Golomb BA, Lawrence DT, Sejnowski TJ. Sexnet: a neural network identifies sex from human faces. In: Lippman R, Touetzky DS, editors. Advances in neural informationprocessing systems 3. San Mateo, CA: Morgan Kauffman 1991. pp. 572-7.

30. Sejnowski TJ, Reingold SC, Kelley DB, Gelperin A. Localization of [3H]-2-deoxyglucose in single molluscan neurones. Nature 1980;287:449-51.

31. Kuffler SW, Sejnowski TJ. Peptidergic and muscarinic excitation at amphibian sympathetic synapses. J Psychol 1982;341:257-78.

32 Sejnowski TJ. What are they projective fields of cortical neurons? In: van Hemmen JL, Sejnowski TJ, editors. 23 Problems in Systems Neuroscience. New York: Oxfor University Press; 2005. pp. 394-405.

33. Sejnowski TJ. AI will make you smarter. In: Brockman J, editor. What to think about machines that think. New York: Harper Perennial; 2015. pp. 118-20.

34. For the first time, a robot passed a medical licensing exam. Available form: https://futurism.com/first-time-robot-passed-medicallicensing-exam [Last accessed on 28 Apr 2020]

35. A bright future for robotic surgery. Available form: https://openmedscience.com/bright-future-for-robotic-surgeons/ [Last accessed on 28 Apr 2020] 